\title{
Kallweit, Nina (2019): Kindliches Erleben von Krieg und Frieden. Eine phänomenographische Untersuchung im politischen Lernen des Sachunterrichts
}

\author{
Springer Spectrum: Wiesbaden
}

\author{
Andrea Becher
}

Eingegangen: 20. September 2020 / Angenommen: 25. September 2020 / Online publiziert: 7. Juli 2021 (C) Der/die Autor(en) 2021

Nina Kallweit befasst sich in ihrer Dissertation mit der Herausforderung, das Erleben von Grundschulkindern zu den Phänomenen ,Krieg ' und ,Frieden ‘ zu erfassen und zu rekonstruieren. Im Rahmen ihres phänomenographisch orientierten Ansatzes eruiert sie einen dichten und umfassenden qualitativen Datensatz und leitet mittels ihrer qualitativ angelegten Analyse und Interpretation Konsequenzen für die Gestaltung von Lehr-Lernprozessen gesellschaftswissenschaftlichen Sachunterrichts ab.

Einleitend legt die Verfasserin umfassend und nachvollziehbar offen, welches Desiderat sie bearbeiten und wie sie sich dabei an dem Forschungsansatz der Phänomenographie orientieren wird als auch, welchen Mehrwert ihre Studie für den Sachunterricht und seine Didaktik birgt. Die Arbeit ist klassisch aufgebaut, in vier Großkapitel gegliedert: Theoretischer Hintergrund, Forschungsmethodologische und lerntheoretische Grundlegungen, Empirische Untersuchung, Fazit und Ausblick.

Im Rahmen des Theoretischen Hintergrunds wird in Kap. 2 (Problemaufriss und Fragestellungen) zunächst der Diskurs um eine Politische Bildung im Sachunterricht der 1970er-Jahre bis zur Gegenwart gelungen nachgezeichnet. Dabei wird ebenso der Forschungsstand zum politischen Lernen/zur Politischen Bildung im Sachunterricht als Zusammenschau dargelegt. Explizit nur Studien aus dem Bereich der Grundschul- und Sachunterrichtsdidaktik fokussierend, wird m.E. jedoch die Chance verpasst, Bezüge zu vergangenen und aktuellen Survey-Studien, die ebenso Kinder zu politischen Aspekten befrag(t)en, herauszustellen. Auch wird nicht darauf verwiesen, wie sich im weiteren Verlauf der Arbeit auf diese Nachzeichnungen bezogen werden kann bzw. soll, bspw. hinsichtlich des eigenen Vorgehens und/oder die Entwicklung von Settingentwürfen.

Prof. Dr. Andrea Becher $(\bowtie)$

Sachunterrichtsdidaktik Lernbereich Gesellschaftswissenschaften, Universität Paderborn, Warburger Str. 100, 33098 Paderborn, Deutschland

E-Mail: andrea.becher@upb.de 
Anschließend begründet Frau Kallweit, Bezug nehmend auf Klafki und vergangene sowie gegenwärtige Diskurse der Friedenserziehung und -pädagogik, passend den von ihr gewählten Themenkontext und benennt u. a. das Desiderat der noch ausstehenden Rekonstruktion von Schülervorstellungen zu Krieg und Frieden im Rahmen des politischen Lernens im Sachunterricht. Nachfolgend wird der Diskurs der Be- und Erforschung von Schülervorstellungen im Kontext der Conceptual ChangeTheorie allgemein gehalten referiert und folgend mit dem für die eigene Arbeit leitenden Ansatz der Phänomenographie hinsichtlich des Potentials einer phänomenografischen Lernforschung in Beziehung gesetzt.

In Kap. 3 (Forschungsstand) trägt die Verfasserin umfassend und sehr dicht die vorhandenen Erkenntnisse aus (inter-)nationalen Forschungen zu Lernvoraussetzungen von Kindern zu ,Krieg ' und ,Frieden“ zusammen und fasst diese, jeweils in drei historische Phasen unterteilt (beginnend mit den 1960er-Jahren), zusammen. Dieses Kapitel bietet den Lesenden einen gelungenen Blick über das Forschungsfeld, wobei jedoch verwundert, dass es vor die fachliche und fachdidaktische Klärung (Kap. 4) der Phänomene Krieg und Frieden gestellt wird. Rekursiv auf die Studienergebnisse (Kap. 3) angewandt, hätten diese Klärungen als Referenzfolie zur Einordnung der empirischen Erkenntnisse über die Lernvoraussetzungen genutzt werden können.

Im nächsten Großkapitel - Forschungsmethodologische und lerntheoretische Grundlegungen -, welches nicht einmal fünf Seiten umfasst, widmet sich Frau Kallweit in Kap. 5 (Qualitative Forschungsansätze) erst ganz allgemein der qualitativen Forschungsweise im Kontext der Sachunterrichtsdidaktik und zeichnet kurz nach, was diese kennzeichnet. Sie setzt diese Klärung dann in Beziehung mit dem Forschungsparadigma des Modells der Didaktischen Rekonstruktion und dieses wiederum mit dem von ihr gewählten Ansatz der Phänomenographie, welchen sie plausibel in das Didaktische Dreieck einordnet.

Vor diesem Hintergrund wird folgend die Phänomenographie schlüssig als favorisierter Forschungsansatz, der insbesondere für didaktisch-fokussierte Forschungsarbeiten einen Mehrwert generiere, begründet. Auf insgesamt 20 Seiten wird der Ansatz der Phänomenographie (Kap. 6) nachvollziehbar und übersichtlich komprimiert zusammengefasst und erläutert, wobei v. a. der Begriff des Erlebens herausgearbeitet wird. Die Verfasserin bezieht sich insbesondere auf die Primärliteratur von Marton und Booth $(1997 ; 2014)$ sowie die für den deutschsprachigen Raum ergiebigen Erläuterungen durch Murmann (2002; 2008), nimmt jedoch leider noch keinen Übertrag auf das eigene Forschungsvorhaben vor.

Dieser Übertrag wird dann aber im nächsten Großkapitel -Empirische Untersuchung - plastisch. Es stellt das Herzstück der Arbeit dar. Einer allgemeinen Darlegung und dann schlüssigen Anwendung der Gütekriterien (Kap. 7) folgen die Ausführungen zur Erhebung und Aufbereitung der Daten (Kap. 8). Gelungen wird die Vorgehensweise der empirischen Arbeit offengelegt, werden das Erhebungsinstrument und die Struktur des Vorgehens schlüssig erläutert und begründet. Der inhaltliche Aufbau der Leitfadeninterviews ist nachvollziehbar, schließt an die bekannten Forschungen an und zeigt, dass ein möglichst alle Aspekte der Phänomene betreffendes Nachzeichnen des Erlebens mitbedacht wurde. Die genutzten Impulse werden ebenso gut begründet und die vorgenommenen Modifizierungen aufgrund des Pretests fußen auf einer nachvollziehbaren kritisch-konstruktiven Reflexion. Die 
Bearbeitung der gewonnenen Daten wird gekonnt offengelegt und erlauben einen intersubjektiver Nachvollzug.

Gleiches gilt für die Datenauswertung (Kap. 9). Dabei orientiert sich die Verfasserin an für die Phänomenographie entwickelten Vorgehensweisen nach Marton und Booth (1997) sowie Dahlgren und Fallsberg (1991). Die Erklärung des Vorgehens ist nachvollziehbar, auch wenn (Anker-)Beispiele aus dem eigenen Datensatz zu einer Art ,Unterfütterung' der eher allgemein gehaltenen Darlegungen schön gewesen wären.

Nach knapp 200 Seiten, in den Kap. 10 und 11, kommt es schließlich zur Darstellung der Ergebnisse des empirischen Erhebungs-, Datenaufbereitungs- und -analyseprozesses. Diese zusammen knapp 135 Seiten umfassenden Kapitel haben es aber in sich! Es werden umfassende Beschreibungskategorien zu den Themenkomplexen Krieg und Frieden geliefert - stets unter- und belegt mit (Anker-)Zitaten aus dem generierten Datensatz. Die sog. Kategoriensätze zu den einzelnen in den Daten eruierten Kategorien sind gelungen und geben den Lesenden einen spannenden und komprimierten Ein- und Überblick in das Erleben und somit mögliche Lernvoraussetzungen der interviewten Kinder. Dabei wird versucht, Hierarchiesierungen vorzunehmen, und - wo dies nicht möglich ist - wird nachvollziehbar begründet, warum eine solche Nachzeichnung mit Hilfe der Daten nicht erfüllbar war. Diese stets enthaltenen und umfassenden Reflexionen zeugen von einem überzeugenden kritisch-konstruktiven Ansatz beim Umgang mit den Daten, sind eine weitere Stärke dieses Kapitel.

Dem Forschungsansatz der Phänomenographie geschuldet, schließen sogleich im Anschluss an die Beschreibungskategorien stets sog. Didaktische Implikationen an, wodurch m. E. jedoch auch Zusammenhänge und Integrationen der einzelnen Kategorien verloren gehen könnten - bspw. im Hinblick auf die Ableitung größer gefasster Didaktischer Implikationen.

In Kap. 12 (Diskussion und Reflexion der Ergebnisse) werden dann die in den Daten eruierten Kategorien nacheinander unter sog. Bezugspunkten zusammengefasst, wobei es zu Abgleichungen mit dem bisherigen Forschungsstand (Kap. 3) kommt. Die Ausführungen lesen sich teilweise wie eine Art Zusammenfassung der eigenen Ergebnisse und es kommt durch den Abgleich zu Wiederholungen der Datenbeschreibungen als auch der Schilderungen zu den bekannten Forschungsergebnissen. Leider werden die empirisch rekonstruierten Erfahrungen der Kinder nur wenig mit den fachwissenschaftlichen Klärungen (Kap. 4) in Beziehung gesetzt, abgeglichen oder eingeordnet.

Da der Ansatz der Phänomenographie insbesondere einen fachdidaktischen Gewinn fokussiert, werden im Rahmen von Kap. 13 (Zusammenführung der Ergebnisse) demnach übergeordnet-leitende Impulse für sachunterrichtliche Lehr-Lernsettings aus den Analysedaten und -ergebnissen abgeleitet. Es liest sich heraus, dass eine Thematisierung von Konflikten favorisiert wird, wobei die Einordnung von sozialen und politischen Konflikten für die Verfasserin essentiell ist.

Dass es auf der Ebene von möglichen Lehr-Lernsettings v. a. um die Bearbeitung und Unterscheidung von Konflikten auf sozialer und gesellschaftlicher und somit auch politischer Ebene geht, wird dann im nächsten und die Arbeit abschließenden Großkapitel - Fazit und Ausblick - noch einmal betont und herausgearbeitet. Frau 
Kallweit kommt zu dem Schluss, dass dafür gerade die Thematisierung von Krieg und Frieden im Kontext eines politischen Lernens im Sachunterricht große Potenziale biete und skizziert weitere Forschungsdesiderate und Anschlussmöglichkeiten.

Insgesamt ist die Arbeit von Frau Kallweit aufgrund der gewählten Thematik und des schlüssigen und überzeugenden Nachzeichnens von Erfahrungskategorien, die die Entwicklung von Materialien zur Thematisierung von Krieg und Frieden mit Grundschulkindern grundlegend leiten können, ein Gewinn für den gesellschaftswissenschaftlichen Sachunterricht und seine Didaktik. Es ist davon auszugehen, dass der Ansatz der Phänomenographie in Zukunft verstärkt für fachdidaktische Forschungszugänge diskutiert werden wird, wobei diese Arbeit dann auch einen grundlegenden Fundus für eine kritisch-konstruktive Einordnung, Diskussion und Reflexion darstellen wird - v. a. auch im Hinblick auf Modifikationen oder die Verwendung in triangulierten Settings bzw. im Kontext von Mixed Methods.

Funding Open Access funding enabled and organized by Projekt DEAL.

Open Access Dieser Artikel wird unter der Creative Commons Namensnennung 4.0 International Lizenz veröffentlicht, welche die Nutzung, Vervielfältigung, Bearbeitung, Verbreitung und Wiedergabe in jeglichem Medium und Format erlaubt, sofern Sie den/die ursprünglichen Autor(en) und die Quelle ordnungsgemäß nennen, einen Link zur Creative Commons Lizenz beifügen und angeben, ob Änderungen vorgenommen wurden.

Die in diesem Artikel enthaltenen Bilder und sonstiges Drittmaterial unterliegen ebenfalls der genannten Creative Commons Lizenz, sofern sich aus der Abbildungslegende nichts anderes ergibt. Sofern das betreffende Material nicht unter der genannten Creative Commons Lizenz steht und die betreffende Handlung nicht nach gesetzlichen Vorschriften erlaubt ist, ist für die oben aufgeführten Weiterverwendungen des Materials die Einwilligung des jeweiligen Rechteinhabers einzuholen.

Weitere Details zur Lizenz entnehmen Sie bitte der Lizenzinformation auf http://creativecommons.org/ licenses/by/4.0/deed.de.

\section{Literatur}

Dahlgren, L.-O., \& Fallsberg, M. (1991). Phenomenography as a qualitative approach in social pharmacy research. Journal of Social and Administrative Pharmacy, 8, (4), 150-156.

Marton, F., \& Booth, S. (1997). Learning and awareness. Mahwah: Lawrence Erlbaum Asscociates.

Marton, F., \& Booth, S. (2014). Lernen und Verstehen. In der Übersetzung von Lydia Murmann und Peter Buck. Berlin: Logis.

Murmann, L. (2002). Physiklernen zu Licht, Schatten und Sehen. Eine phänomenographische Untersuchung in der Primarstufe. (Studien zum Physiklernen; Bd. 24), Berln: Logos.

Murmann, L. (2008). Phänomenographie und Didaktik. In M. A. Meyer, M. Prenzel \& S. Hellekamps (Hrsg.), Perspektiven der Didaktik (S. 187-199). (Zeitschrift für Erziehungswissenschaft; Sonderheft 9), Wiesbanden: VS. 